\title{
A Case of Stereotactic Radiation in Skull Base Solitary Fibrous Tumor: More Harm Than Good?
}

\author{
Peter L. Santa Maria ${ }^{1}$ Waleed M. Abuzeid ${ }^{1} \quad$ Jayakar V. Nayak ${ }^{1}$ Steven D. Chang ${ }^{2} \quad$ Nikolas H. Blevins ${ }^{1}$ \\ ${ }^{1}$ Department of Otolaryngology, Head and Neck Surgery, Stanford \\ University Hospital and Clinics, Stanford, California, United States \\ ${ }^{2}$ Department of Neurosurgery, Head and Neck Surgery, Stanford \\ University Hospital and Clinics, Stanford, California, United States \\ Address for correspondence Peter Luke Santa Maria, MBBS, PhD, \\ Department of Otolaryngology, Head and Neck Surgery, Stanford \\ University Hospital and Clinics, 801 Welch Rd, Stanford, CA 94305, \\ United States (e-mail: psantamaria@ohns.stanford.edu).
}

J Neurol Surg Rep 2014;75:e214-e216.

\begin{abstract}
Objective Due to its location, total resection of a skull base solitary fibrous tumor (SFT) can lead to morbidity with injury to lower cranial nerves, and a decision must be made between subtotal resection with possible stereotactic radiotherapy or total resection with cranial nerve morbidity. We report the long-term outcomes and review the literature of a case of stereotactic radiation in SFT to provide evidence for making this decision.

Design A retrospective case review.

Setting An academic tertiary referral center.

Results We present a case with $>10$ years follow-up of radiation following skull base SFT, initially misdiagnosed as schwannoma, where radiotherapy did not improve recurrence or metastatic behavior and led to complications during subsequent surgical resection.

Conclusions SFT often masquerades as schwannoma, especially in the skull base. Careful immunohistochemistry, including CD34 expression, is critical to the diagnosis and

\section{Keywords}

- solitary fibrous tumor

- stereotactic radiation management. This case highlights that total tumor resection of SFT remains the gold standard of treatment. Stereotactic radiation is not recommended in the management of skull base SFT.
\end{abstract}

\section{Introduction}

Solitary fibrous tumor (SFT) is a neoplasm that mimics other skull base lesions. ${ }^{1}$ We present a case of malignant regional metastasis of SFT, initially misdiagnosed as schwannoma, occurring $>10$ years after treatment with stereotactic radiation. Stereotactic radiation combined with subtotal resection is often considered when total tumor resection is likely to lead to cranial nerve morbidity. This patient's poor outcome questions the utility of radiation for skull base SFT because such treatment may fail to obtain long-term growth control and increases the potential for future complications. This case highlights the importance of obtaining the correct diagnosis, achieving total resection, and the need for lifetime follow-up of SFT.

\section{Case Material}

A 41-year-old man presented to our institution with 1 month of progressive right facial nerve weakness. He initially presented 15 years ago with headaches and progressive hypoglossal palsy and was diagnosed with what was believed to be a right jugular foramen schwannoma at an overseas institution. He underwent subtotal resection via a retrosigmoid approach. The remnant was stable for 3 years until interval growth was noted upon follow-up at an outside institution in
License terms Stuttgart · New York

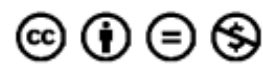

March 15, 2014 accepted after revision June 8, 2014 published online August 11, 2014
DOI http://dx.doi.org/ 10.1055/s-0034-1387196. ISSN 2193-6358. 
the United States. Consequently, the patient underwent a revision subtotal resection 11 years ago, which was complicated by postoperative glossopharyngeal and vagus nerve palsies. With the impression that the tumor was still a schwannoma, postoperative adjunctive treatment using gamma knife stereotactic radiation was administered (32 Gy, single fraction).

Interval magnetic resonance imaging surveillance postradiation showed no growth of the skull base remnant until the most recent presentation, now 11 years later ( - Fig. 1). At his most recent preoperative presentation he had cranial nerve VII (House-Brackmann grade 2), IX, X, and XII palsy with moderate sensorineural hearing loss with $100 \%$ word recognition on the
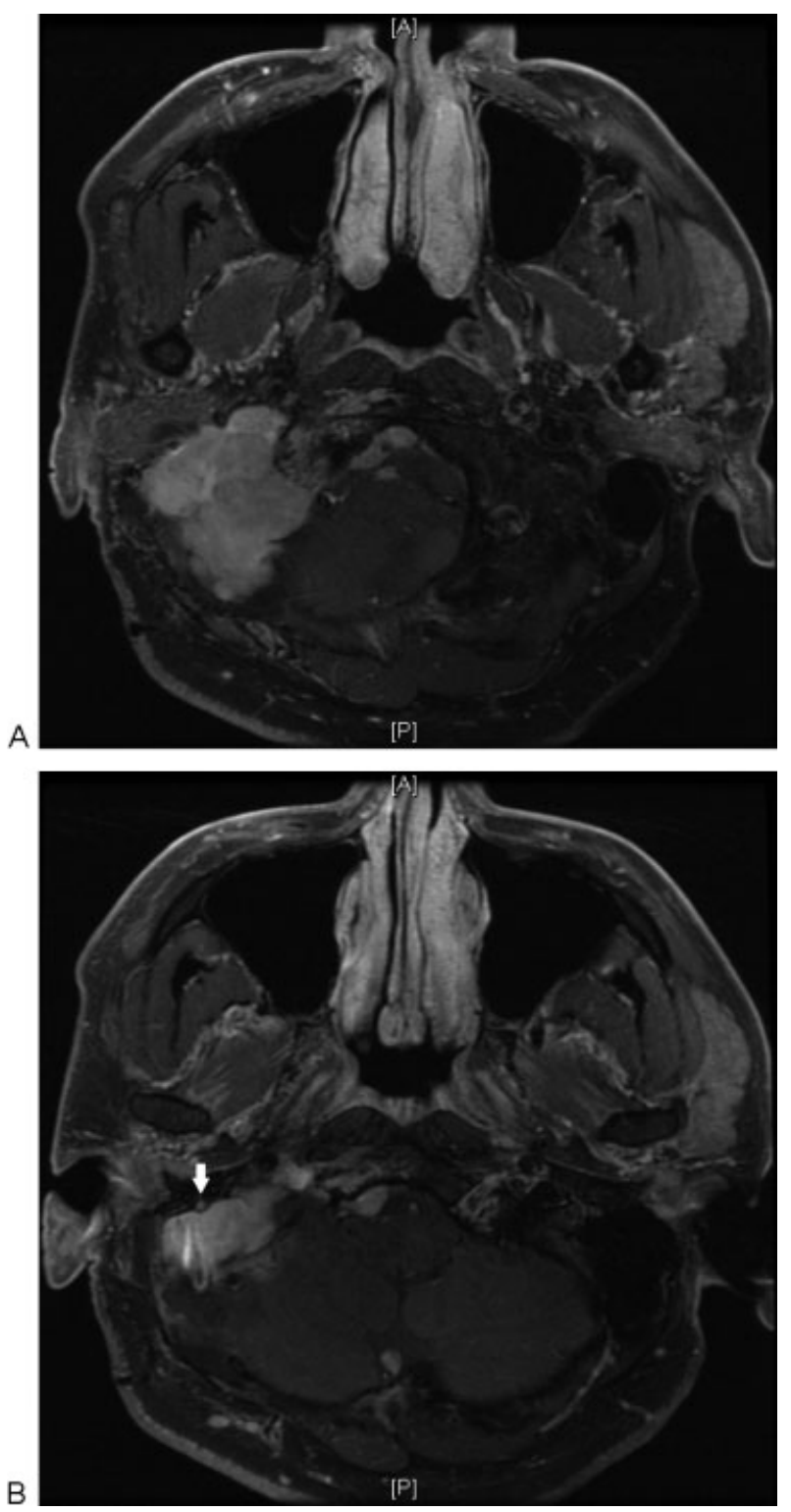

Fig. 1 The T1-weighted postgadolinium contrast features of solitary fibrous tumor (SFT) as seen in the most recent presentation. The tumor is based around the right jugular foramen, with (A) a small intradural component seen along the clivus and (B) abutting the vertical facial nerve (white arrow). The magnetic resonance imaging features of SFT in this location are indistinguishable from schwannoma. affected side. Due to the accelerated growth and new onset of right facial nerve palsy, additional surgery via a right transjugular approach was advocated. Intraoperatively, the tissue was fibrous, well circumscribed, multilobulated, and rubbery. The facial nerve was compressed at the stylomastoid foramen but was not invaded. Frozen section diagnosis by an experienced head and neck pathologist suggested "non-aggressive fibrous tumor without features of schwannoma" but was unable to give a specific diagnosis. The resection of the extradural component was completed but, given the uncertain diagnosis and risk of more aggressive resection, a small intradural component was not removed.

The final pathology was reported as a solitary fibrous tumor. This was based on spindle cell features including plump ovoid cells with limited cytoplasm haphazardly arranged in a rich collagen background, diffusely positive for CD34 and negative for both S-100 and smooth muscle actin. The historic pathology from the outside institution was obtained and reexamined using immunohistochemistry to confirm that this was also SFT. On further work-up of the patient, a right level 2 lymph node was noted to be enlarged at $2 \mathrm{~cm}$. Fine-needle aspiration confirmed metastatic SFT given the presence of spindle cell features.

The case was discussed at a multidisciplinary skull base conference where an anterior approach was favored to address the residual intradural component. The patient underwent endonasal transclival total resection of the intradural SFT component with brainstem decompression. This was complicated by a vigorous cerebrospinal fluid (CSF) leak requiring multiple revision procedures. Postoperatively, a vertebrobasilar vasospasm of unknown etiology developed, necessitating ipsilateral vertebral artery balloon dilation and nicardipine infusion. This episode resulted in a small medullary ischemic cerebrovascular accident for which the patient is currently undergoing rehabilitation. He will ultimately require a neck dissection.

\section{Discussion}

SFT can be benign or malignant, with the only differentiating factor often the presence of metastasis. It is typically well defined and nonencapsulated with alternating hyper- and hypocellular regions of spindle cells on a collagen background. SFT is most commonly found in the pleura, but other sites include the lung, liver, breast, meninges, pelvis, sinonasal cavity, salivary glands, and orbit. ${ }^{2}$ Skull base SFT is usually a metastatic lesion, often from the liver, but it may arise primarily. ${ }^{3}$ Skull base SFT differs from other sites in that it is presents in females with a wide age distribution. ${ }^{2}$ Once diagnosed with SFT, a full-body positron emission tomography/computed tomography should be performed to determine whether this is a primary or metastatic lesion and also to stage the disease, looking for subclinical metastasis.

In this case, the misdiagnosis as schwannoma led to the decision to perform a subtotal resection. Had it been correctly identified as SFT, given the morbidity to the lower cranial nerves of a total resection, it can be attractive to perform a subtotal resection and treat with stereotactic radiation. The 
Table 1 Antigens that differentiate three histologically similar tumors of the jugular foramen ${ }^{\mathrm{a}}$

\begin{tabular}{|l|l|l|l|}
\hline \multirow{2}{*}{ Tumor } & \multicolumn{3}{l|}{ Antigen } \\
\cline { 2 - 4 } & CD34 & S-100 & EMA \\
\hline Solitary fibrous tumor & + & - & + \\
\hline Meningioma & - & - & - \\
\hline Schwannoma & - & + & - \\
\hline
\end{tabular}

Abbreviations: +, positive staining; -, negative staining; CD34, cluster of differentiation 34; EMA, epithelial membrane antigen.

${ }^{\mathrm{a}} \mathrm{CD} 34, \mathrm{~S}-100$, and EMA staining can be assessed as discriminating features.

aggressive behavior of SFT, namely multiple recurrences and metastasis found in 13 to $23 \%{ }^{2}$ is predicted based on histology. This behavior is more often associated with high mitotic activity, nuclear atypia, hypercellularity, or necrosis but can occur, as in this case, without any of these. ${ }^{4}$ This highlights the importance of total surgical resection for SFT, even with seemingly benign histologic and imaging features.

The preoperative diagnosis of SFT, via imaging, is difficult with no hallmark radiologic features. ${ }^{1}$ The pathologic appearance of schwannoma, meningioma, and solitary fibrous tumor can overlap and necessitate immunohistochemical distinction via CD34, S-100 and epithelial membrane antigen $^{2}$ (-Table 1). SFT most commonly stains for vimentin, BCL2, and CD99 but is usually differentiated via positive staining for CD34. ${ }^{1}$ Subtotal resection is acceptable for schwannoma; however, in SFT it is a risk factor for recurrence and metastasis. ${ }^{2}$

The case presented is the longest reported follow-up of a patient with extrathoracic SFT receiving radiation based on review of the English literature. It allows us to look back at the utility of stereotactic radiation in skull base SFT when the literature is lacking long-term follow-up in this area. Extrathoracic SFT can recur and have distant metastasis up to 25 years after primary treatment. ${ }^{1}$ The only large series of SFT reporting radiation have limited follow-up and reported no difference in survival with radiation in thoracic SFT at 5 years ${ }^{5}$ and questionable benefit in central nervous system SFT ${ }^{1}$ with most cases showing local growth and metastasis despite radiation. Case reports claiming successful treatment of SFT with radiation must be interpreted cautiously because follow-up is often limited and SFT can lay indolent before becoming aggressive without radiation. There are isolated cases of adjuvant paclitaxel being used in pelvic SFT, ${ }^{6}$ but no long-term follow-up has been reported, and the efficacy of adjuvant chemotherapy is not known. Because of the unrelenting nature of SFT, it is difficult to determine whether radiation exposure contributed to the malignant behavior or this represented natural progression. CSF leak rates are increased in transsphenoidal surgery following radiation ${ }^{7}$ and this was potentially a factor in the patient's poor outcome. Occlusive radiation vasculopathy secondary to radiation $^{8}$ may have also played a role in the delayed vertebrobasilar artery spasm leading to focal medullary ischemia.

\section{Conclusions}

SFT with malignant behavior can masquerade as several other benign and malignant skull base tumors, and careful immunohistochemistry, including CD34 expression, is critical to the diagnosis and treatment planning. Complete resection of SFT is advocated because these lesions may recur over long periods. Radiation treatment in SFT should not substitute for total tumor resection. It is not recommended because it has questionable benefit and may not positively influence the tumor behavior while possibly complicating subsequent surgical resection.

\section{Acknowledgments}

We thank the Garnett Passe and Rodney Williams Memorial Foundation for their support.

\section{Conflicts of Interest}

The authors have nothing to disclose.

\section{References}

1 Bisceglia M, Galliani C, Giannatempo G, et al. Solitary fibrous tumor of the central nervous system: a 15-year literature survey of 220 cases (August 1996-July 2011). Adv Anat Pathol 2011;18(5): 356-392

2 Deniz K, Kontas O, Tucer B, Kurtsoy A. Meningeal solitary fibrous tumor: report of a case and literature review. Folia Neuropathol 2005;43(3):178-185

3 Ogawa K, Tada T, Takahashi S, et al. Malignant solitary fibrous tumor of the meninges. Virchows Arch 2004;444(5):459-464

4 Gessi M, Gielen GH, Roeder-Geyer ED, et al. Extracranial metastasizing solitary fibrous tumors (SFT) of meninges: histopathological features of a case with long-term follow-up. Neuropathology 2013;33(1):68-74

5 Milano MT, Singh DP, Zhang H. Thoracic malignant solitary fibrous tumors: a population-based study of survival. J Thorac Dis 2011; 3(2):99-104

6 Kawamura S, Nakamura T, Oya T, et al. Advanced malignant solitary fibrous tumor in pelvis responding to radiation therapy. Pathol Int 2007;57(4):213-218

7 Nishioka H, Haraoka J, Ikeda Y. Risk factors of cerebrospinal fluid rhinorrhea following transsphenoidal surgery. Acta Neurochir (Wien) 2005;147(11):1163-1166; discussion 1166

8 Zou WX, Leung TW, Yu SC, et al. Angiographic features, collaterals, and infarct topography of symptomatic occlusive radiation vasculopathy: a case-referent study. Stroke 2013;44(2): 401-406 